\title{
Generalised Sasa-Satsuma Equation: Densities Approach to New Infinite Hierarchy of Integrable Evolution Equations
}

https://doi.org/10.1515/zna-2018-0377

Received August 7, 2018; accepted September 23, 2018; previously published online October 16, 2018

\begin{abstract}
We derive the new infinite Sasa-Satsuma hierarchy of evolution equations using an invariant densities approach. Being significantly simpler than the Lax-pair technique, this approach does not involve ponderous $3 \times 3$ matrices. Moreover, it allows us to explicitly obtain operators of many orders involved in the time evolution of the Sasa-Satsuma hierarchy functionals. All these operators are parts of a generalised Sasa-Satsuma equation of infinitely high order. They enter this equation with independent arbitrary real coefficients that govern the evolution pattern of this multiparameter dynamical system.
\end{abstract}

Keywords: Evolution Equations; Integrable System; Sasa-Satsuma.

\section{Introduction}

An integrable hierarchy is an infinite sequence of partial differential equations that starts with a particular case. The starting equation could be the Korteweg-de Vries (KdV) equation [1, 2], nonlinear Schrödinger equation (NLSE) [3], Toda lattice [4], certain classes of Painlevé equations [5], etc. Each successive equation in the hierarchy normally takes a more complex form than the previous one. An integrable hierarchy can be considered as a system of commuting Hamiltonian flows [6]. An infinite number of commuting flows can be obtained recursively. Most of

\footnotetext{
*Corresponding author: A. Ankiewicz, Optical Sciences Group, Research School of Physics and Engineering, The Australian National University, Canberra, ACT 2601, Australia, E-mail: Adrian.Ankiewicz@anu.edu.au

U. Bandelow: Weierstrass Institute for Applied Analysis and Stochastics, Mohrenstraße 39, 10117 Berlin, Germany

N. Akhmediev: Optical Sciences Group, Research School of Physics and Engineering, The Australian National University, Canberra, ACT 2601, Australia
}

the works on integrable hierarchies are written by mathematicians and may not be easily accessible. Consequently, purely mathematical results may not be easily used in practical applications. Even if recursive rules for deriving higher-order equations of a particular hierarchy are provided, obtaining explicit forms of these equations is a different matter. A countable number of these equations have been presented so far. Moreover, some of these hierarchies remain unknown because of obscurities in the definition of "integrability". The Painlevé criterion can be useful in finding these special cases [7]. However, using it does not exclude the possibility that another integrable case, not covered by the technique, remains hidden. In mathematical terms, the Painlevé criterion is a necessary but not sufficient condition for finding integrable equations.

One important observation is that members of the hierarchy can be written in the form of one general equation, which combines all individual equations into a single one $[8,9]$. This general equation can have an infinite number of operators controlling the time evolution of a system [8, 9]. It includes all equations of the hierarchy as particular cases with arbitrary real coefficients, which govern the contribution of each operator to the whole. The convenience of such representation lies in the arbitrariness of these coefficients. When all of them are zero except one, we obtain an individual equation of the hierarchy. Having two or more coefficients being nonzero provides more complicated equations that can be of interest due to the special case in physics that such an equation can describe. One example is the Heisenberg spin chain dynamics [7].

Such general equations could be of great importance for physics because higher-order terms in this equation may describe finer effects such as higher-order dispersion or higher-order nonlinearities in wave propagation phenomena. They become important only beyond the basic approximation that is usually described by the lowestorder equation. The brightest example of such approach is soliton science, which started with the KdV equation [10] and NLSE [11]. Clearly, the basic properties of solitons have to be known before we can move to such 
elaborations as pulse compression [12], self-frequency shift [13], wave breaking [14], etc. Particular cases of such step-by-step improvements in wave description are well known in optics. Starting from simple theory [15], the soliton approach has been advanced with the contributions of higher-order terms in later works [16-18]. Similar "upgrades" of the soliton approach [19] have been provided in water wave theory [20-22]. Higher-order equations are no less important in the theory of rogue waves. Several equations admitting rogue waves have been presented so far. In addition to the NLSE, we can mention coupled three-wave equations [23], vector NLSEs [24], Davey-Stewartson equations [25, 26], evolution equations in quadratic media [27], Kadomtsev-Petviashvili equation [28], etc. Extensions to higher-order versions can be expected for integrable cases.

Unfortunately, not all higher-order terms in these upgrades result in integrable equations. A specific set of coefficients is required for these very special cases. It is indeed fortunate when such an "upgrade" belongs to a general equation of an integrable hierarchy. The chances are low if there is only one hierarchy that starts with the given base equation. Finding new hierarchies is thus an important task, which may significantly improve the accuracy of modelling physical phenomena. Luckily, there are at least two "general equations" that have the NLSE as a base. One of these is a Hirota hierarchy $[8,9]$, while the other one, found recently [29], is a Sasa-Satsuma hierarchy. Both start with the NLSE as the base evolution equation. Thus, both of them could be called NLSE hierarchies. In order to avoid confusion and distinguish them explicitly, we label them here as the generalised Hirota and generalised Sasa-Satsuma equations (SSEs).

The first few equations of the Hirota hierarchy are the NLSE [11], the third-order Hirota equation [30], the fourthorder Lakshmanan-Porsezian-Daniel (LPD) equation [31], and the quintic extension of this sequence [32, 33]. Higherorder extensions (to sixth-order, etc.) have been presented in explicit forms in $[8,9]$. On the other hand, the two starting equations of the Sasa-Satsuma hierarchy are the NLSE and the third-order SSE [34-36]. Higher-order extensions (fourth-order, etc.) have been discovered in [29].

In the present work, we make further progress in dealing with the general SSE. Namely, we derive many equations in the hierarchy using invariant densities. This approach is significantly simpler than the original Lax pair technique developed in [29]. It does not require operations with cumbersome $3 \times 3$ matrices. Moreover, recurrent relations in this technique are straightforward and require only the knowledge of the infinite set of invariant densities $H_{j}$. These can be found by representing any given evolution equation in the form of continuity equations:

$$
\frac{\partial H_{j}}{\partial t}+\frac{\partial J_{j}}{\partial x}=0
$$

of various orders $j$, where $J_{j}$ is the corresponding invariant flow of the order $j$. These continuity equations can be constructed in the same way as for the NLSE [37]. This process requires nothing more than purely algebraic transformations. For the SSE in the form:

$$
i u_{t}+\frac{u_{x x}}{2}+|u|^{2} u=i \varepsilon\left[u_{x x x}+3\left(|u|^{2}\right)_{x} u+6|u|^{2} u_{x}\right],
$$

for the first order, we have

$$
H_{1}=|u|^{2}
$$

and

$$
J_{1}=\frac{i}{2}\left(u u_{x}^{*}-u^{*} u_{x}\right)-\varepsilon\left[\left(|u|^{2}\right)_{x x}-4\left|u_{x}\right|^{2}+6|u|^{4}\right] .
$$

When the free real parameter $\varepsilon=0$, these densities are the same as for the NLSE [37]. The whole infinite set of higher-order invariant densities and flows can be found easily. An alternative way of obtaining the invariant densities is using the generating function technique [38]. Below, we consider the invariant densities to be known. As we can see from Section 2, with this approach, the whole set of calculations occupies only one page. Integrability of the higher-order equations is guaranteed, as it is based on the invariants of the SSE.

Our present technique confirms that all terms in the SSE hierarchy found in [29] are correct, and it provides an independent and simple way of working with these highly nontrivial extensions of the NLSE and SSE. Again, we stress that the general equation related to this hierarchy contains an infinite number of real parameters. The practical benefit of such approach is that this general equation contains higher-order terms with adjustable coefficients, and these could describe wave propagation phenomena with higher accuracy.

\section{Derivation of Generalised Sasa-Satsuma Equation from Invariant Densities}

As noted above, the results of our first article [29] can be derived using an even simpler technique that uses invariant densities of the SSE. This can be done in a way that is related to that used in [9] for the Hirota (previously called NLSE) hierarchy. The difference lies in using the set of 
invariant densities of the SSE instead of invariant densities of the NLSE. Let us write the hierarchy, containing an infinite set of equations, in the form:

$$
i u_{t}+\sum_{n=1}^{\infty}\left(\alpha_{2 n} S_{2 n}-i \alpha_{2 n+1} S_{2 n+1}\right)=0,
$$

where $S_{j}$ is the functional of the order $j$ for the envelope function $u(x, t)$, and $\alpha_{j}$ are arbitrary real coefficients. We stress that the coefficients $\alpha_{j}$ are not small parameters. They are finite real numbers, thus making our approach far from being just another perturbation analysis. In (2), we explicitly separated even and odd terms for the reason that will be clear in the following.

The SSE functional can be written in the form:

$$
S_{3}=\frac{6}{b^{2}} u_{x}|u|^{2}+\frac{3}{b^{2}} u\left(|u|^{2}\right)_{x}+u_{x x x} .
$$

Other forms [34-36] follow from (3) after simple transformations. The inclusion of the arbitrary real constant $b$ in (3) follows from free scaling on the variable $x$. In the normalisation of [29], $b=1$, but here we find it more appropriate to use $b=\sqrt{2}$.

The first density of the integral invariant of the SSE is

$$
H_{1}=|u|^{2} .
$$

The transverse integral (i.e. over $x$ ) of this expression represents the conserved energy during evolution.

We define the variational (Frechet) derivative as

$$
\mathcal{F} \equiv \frac{\partial}{\partial u^{*}}-\frac{\partial}{\partial x} \frac{\partial}{\partial u_{x}^{*}}+\frac{\partial^{2}}{\partial x^{2}} \frac{\partial}{\partial u_{x x}^{*}}-\cdots
$$

The second invariant density (integrand) of the SSE, divided by $b$, is

$$
H_{2}=-\frac{1}{2}\left[u_{x} u^{*}+u u_{x}^{*}\right] .
$$

The Frechet derivative of $\mathrm{H}_{2}$ is zero.

The third invariant density of the SSE divided by $b^{2}$ is

$$
H_{3}=\frac{u^{*} u_{x x}}{2}+\frac{u u^{*}{ }_{x x}}{2}+\frac{2}{b^{2}}|u|^{4} .
$$

Its integral with respect to $x$ is the Hamiltonian.

Taking the variational derivative produces the NLS term:

$$
\mathcal{F}\left(H_{3}\right)=S_{2}=\frac{4}{b^{2}} u|u|^{2}+u_{x x} .
$$

We now take $b=\sqrt{2}$ to get the usual form of the NLSE, $i u_{t}+\alpha_{2} S_{2}=0$, i.e.

$$
i u_{t}+\alpha_{2}\left(2 u|u|^{2}+u_{x x}\right)=0 .
$$

Note that this normalisation matches the form used in [9], $K_{2}=2 u|u|^{2}+u_{x x}$.

The fourth invariant of the SSE, divided by $b^{3}$, is

$$
\begin{aligned}
H_{4}= & -\frac{1}{2}\left[\frac{10}{b^{2}} u_{x} u\left(u^{*}\right)^{2}+u_{x x x} u^{*}\right. \\
& \left.+\frac{10}{b^{2}} u^{2} u^{*}\left(u^{*}\right)_{x}+u\left(u^{*}\right)_{x x x}\right] .
\end{aligned}
$$

The Frechet derivative of $\mathrm{H}_{4}$ is also zero.

The invariant density $H_{5}$ of the SSE, divided by $b^{4}$ is:

$$
\begin{aligned}
H_{5}= & \frac{8|u|^{6}}{b^{4}}+\frac{4 u^{2}\left(u_{x}^{*}\right)^{2}}{b^{2}}+\frac{4\left(u^{*}\right)^{2} u_{x}^{2}}{b^{2}} \\
& +\frac{14|u|^{2}\left|u_{x}\right|^{2}}{b^{2}}+\frac{7 u^{*}|u|^{2} u_{x x}}{b^{2}} \\
& +\frac{7 u|u|^{2} u_{x x}^{*}}{b^{2}}+\frac{u^{*} u_{x x x x}}{2}+\frac{u u^{*}{ }_{x x x x}}{2}
\end{aligned}
$$

Setting $b^{2}=2$, we get

$$
\begin{aligned}
H_{5}= & 2 u^{2}\left(u_{x}^{*}\right)^{2}+2\left(u^{*}\right)^{2} u_{x}^{2}+7|u|^{2}\left|u_{x}\right|^{2} \\
& +\frac{7}{2}|u|^{2} u^{*} u_{x x}+\frac{u^{*} u_{x x x x}}{2} \\
& +\frac{u u^{*}{ }_{x x x x}}{2}+2|u|^{6}+\frac{7}{2}|u|^{2} u u_{x x}^{*} .
\end{aligned}
$$

We obtain the higher-order terms by using the operator $\mathcal{F}$ from (5). Thus,

$$
\mathcal{F}\left(H_{5}\right)=S_{4} .
$$

The fourth-order functional is thus

$$
\begin{aligned}
S_{4}= & \frac{24}{b^{4}}|u|^{4} u+\frac{6}{b^{2}} u^{2} u_{x x}^{*}+\frac{12}{b^{2}} u\left|u_{x}\right|^{2} \\
& +\frac{14}{b^{2}}|u|^{2} u_{x x}+\frac{8}{b^{2}} u^{*} u_{x}^{2}+u_{x x x x} .
\end{aligned}
$$

Then, the functional (13) resembles the one in the LPD equation [9] with the form of the terms but has different coefficients in front of each of them.

The sixth invariant is

$$
\begin{aligned}
H_{6}= & -\frac{44\left(u^{*}\right)^{2} u^{3} u_{x}^{*}}{b^{4}}-\frac{14\left(u^{*}\right)^{2} u_{x} u_{\mathrm{xx}}}{b^{2}} \\
& -\frac{11 u^{*} u_{x}^{2} u_{x}^{*}}{b^{2}}-\frac{u^{*} u_{5 x}}{2} \\
& -u^{2}\left[\frac{44\left(u^{*}\right)^{3} u_{x}}{b^{4}}+\frac{14 u_{x}^{*} u_{x x}^{*}}{b^{2}}+\frac{9 u^{*} u^{*}{ }_{x x x}}{b^{2}}\right]
\end{aligned}
$$




$$
\begin{aligned}
& -u\left[\frac{24 u^{*} u_{x} u_{x x}^{*}}{b^{2}}+\frac{24 u^{*}\left(u_{x}\right)^{*} u_{x x}}{b^{2}}+\frac{9\left(u^{*}\right)^{2} u_{x x x}}{b^{2}}\right. \\
& \left.+\frac{11 u_{x}\left(u_{x}^{*}\right)^{2}}{b^{2}}+\frac{u_{5 x}^{*}}{2}\right]
\end{aligned}
$$

The Frechet derivative of $H_{6}$ is also zero.

The seventh invariant density in the set is

$$
\begin{aligned}
H_{7}= & \frac{1}{2}\left\{u \left[\frac{u^{*}}{b^{2}}\left(73 u_{x} u_{x x x}^{*}+98 u_{x x} u_{x x}^{*}+73 u_{x}^{*} u_{x x x}\right)\right.\right. \\
& +\frac{97}{b^{2}}\left|u_{x}\right|^{2} u_{x x}^{*}+\frac{194}{b^{4}}\left(u^{*}\right)^{3} u_{x}^{2} \\
& \left.+\frac{22}{b^{2}}\left(u^{*}\right)^{2} u_{x x x x}+u_{6 x}^{*}+\frac{45}{b^{2}}\left(u_{x}^{*}\right)^{2} u_{x x}\right] \\
& +u^{*}\left\{\frac{u_{x}}{b^{2}}\left[43 u^{*} u_{x x x}+97 u_{x}^{*} u_{x x}\right]\right. \\
& \left.+\frac{45}{b^{2}} u_{x}^{2} u_{x x}^{*}+\frac{29}{b^{2}}\left(u^{*}\right)^{2} u_{x x}^{2}+u_{6 x}\right\} \\
& +\frac{80}{b^{6}}|u|^{8}+2 u^{3} \frac{u^{*}}{b^{4}}\left[76 u^{*} u_{x x}^{*}+97\left(u_{x}^{*}\right)^{2}\right] \\
& +u^{2}\left[\frac{43}{b^{2}} u_{x}^{*} u_{x x x}^{*}+\frac{572}{b^{4}}\left(u^{*}\right)^{2}\left|u_{x}\right|^{2}+\frac{152}{b^{4}}\left(u^{*}\right)^{3} u_{x x}\right. \\
& \left.\left.+\frac{29}{b^{2}} u^{2}\left(u_{x x}^{*}\right)^{2}+\frac{22}{b^{2}} u^{*} u_{x x x x}^{*}\right]\right\} .
\end{aligned}
$$

We again obtain the sixth-order functional by taking the variational derivative of $H_{7}$ :

$$
\begin{aligned}
S_{6}= & u_{6 x}+\frac{55}{b^{4}} u^{3}\left(u_{x}^{*}\right)^{2}+\frac{45}{b^{2}} u_{x}^{2} u_{x x}^{*}+\frac{32}{b^{2}} u u_{x} u_{x x x}^{*} \\
& +\frac{43}{b^{2}} u^{*} u_{x} u_{x x x}+\frac{175}{b^{4}}\left(u^{*}\right)^{2} u u_{x}^{2}+\frac{53}{b^{2}} u u_{x x} u_{x x}^{*} \\
& +\frac{31}{b^{2}} u^{*} u_{x x}^{2}+\frac{20}{b^{2}}|u|^{2} u_{x x x x}+\frac{160}{b^{6}}|u|^{6} u \\
& +\frac{110}{b^{4}} u^{*} u^{3} u_{x x}^{*}+\frac{330}{b^{4}} u^{*} u^{2}\left|u_{x}\right|^{2}+\frac{170}{b^{4}}|u|^{4} u_{x x} \\
& +\frac{8}{b^{2}} u^{2} u_{x x x x}^{*}+\frac{95}{b^{2}}\left|u_{x}\right|^{2} u_{x x}+\frac{37}{b^{2}} u\left(u_{x}\right)^{*} u_{x x x} .
\end{aligned}
$$

Clearly, this process can be continued indefinitely, generating the infinite hierarchy of operators of the general SSE (2).

\subsection{Operators $S_{j}$ with Normalisation $b^{2}=2$}

In conclusion, we can give explicitly the general (2). We use $b^{2}=2$ throughout. The lowest-order functional $S_{2}[u(x, t)]$ in (2) is given by

$$
S_{2}=u_{x x}+2 u|u|^{2}
$$

while (3) gives

$$
S_{3}=3 u_{x}|u|^{2}+\frac{3}{2} u\left(|u|^{2}\right)_{x}+u_{x x x} .
$$

Using (13), we obtain

$$
\begin{aligned}
S_{4}= & 6|u|^{4} u+3 u^{2} u_{x x}^{*}+6 u\left|u_{x}\right|^{2} \\
& +7|u|^{2} u_{x x}+4 u^{*} u_{x}^{2}+u_{x x x x} .
\end{aligned}
$$

Renormalising the form given in [29], we obtain

$$
\begin{aligned}
S_{5}= & u_{5 x}+20|u|^{4} u_{x}+\frac{5}{2} u^{2} u_{x x x}^{*} \\
& +\frac{25}{2} u\left(u_{x} u_{x x}^{*}+u_{x}^{*} u_{x x}\right)+10|u|^{2} u^{2} u_{x}^{*} \\
& +10\left|u_{x}\right|^{2} u_{x}+\frac{15}{2}|u|^{2} u_{x x x}+15 u^{*} u_{x} u_{x x} .
\end{aligned}
$$

Using (15), we obtain

$$
\begin{aligned}
S_{6}= & \frac{55}{4} u^{3}\left(u_{x}^{*}\right)^{2}+\frac{45}{2} u_{x}^{2} u_{x x}^{*}+16 u u_{x} u_{x x x}^{*} \\
& +\frac{43}{2} u^{*} u_{x} u_{x x x}+\frac{175}{4}\left(u^{*}\right)^{2} u u_{x}^{2}+ \\
& +\frac{53}{2} u u_{x x} u_{x x}^{*}+\frac{31}{2} u^{*} u_{x x}^{2}+10|u|^{2} u_{x x x x} \\
& +20|u|^{6} u+\frac{55}{2} u^{*} u^{3} u_{x x}^{*} \\
& +\frac{165}{2} u^{*} u^{2}\left|u_{x}\right|^{2}+\frac{85}{2}|u|^{4} u_{x x}+4 u^{2} u_{x x x x}^{*} \\
& +\frac{95}{2}\left|u_{x}\right|^{2} u_{x x}+\frac{37}{2} u\left(u_{x}\right)^{*} u_{x x x}+u_{6 x} .
\end{aligned}
$$

\section{Reduction to Real-Valued Forms for Odd Terms Only}

For the moment, let us restrict ourselves to the odd-order functionals only. If we additionally specify that the functions $u(x, t)$ should be real valued, then (2) reduces to higher-order forms of the modified $\mathrm{KdV}(\mathrm{mKdV})$ equation. In this case, (17) reduces to the basic mKdV equation,

$$
u_{t}-\alpha_{3} S_{3}=0
$$


with

$$
M_{3}=u_{x x x}+6 u_{x} u^{2}
$$

For the fifth order, we obtain

$$
u_{t}-\alpha_{5} S_{5}=0 \text {, }
$$

from (18):

$$
M_{5}=u_{5 x}+10\left(u_{x x x} u^{2}+4 u u_{x} u_{x x}+u_{x}^{3}+3 u_{x} u^{4}\right) .
$$

The basic soliton solution of the SSE hierarchy with odd terms only, viz.

$$
u_{t}-\alpha_{3} S_{3}-\alpha_{5} S_{5}-\cdots=0,
$$

is

$$
u=p \operatorname{sech}[p(x+s t)]
$$

where $s=\sum_{n=1}^{\infty} \alpha_{2 n+1} p^{2 n}$ represents a velocity. Equation (24) is real, and in this case, the SSE and its basic solution are the same as for the lowest-order mKdV equation, viz.

$$
u_{t}+a_{3}\left(u_{x x x}+6 u^{2} u_{x}\right)=0 .
$$

The general (23), with odd-numbered functionals only, is essentially the mKdV hierarchy [39] of equations. Thus, it is a (real) subset of the Sasa-Satsuma hierarchy. We expect the form of the solution (24) to be valid for all these equations. Moreover, if $u(x, t)$ is an mKdV solution, then scaling shows that $u^{\prime}=q u\left(q x, q^{3} t\right)$ is also its solution for any real $q$, so we can just set $p=1$ in the above, without loss of generality.

\section{Relations between SSE, Hirota (NLS), and mKdV Hierarchies}

To find the relation between SSE, Hirota (NLS), and mKdV hierarchies, we need a consistent normalisation and use $b=\sqrt{2}$ in the results found above; we note that this is different from that used in the earlier article [29]. We denote our previously found [9] functionals for the Hirota (NLS) hierarchy by $K_{j}$, so that the equations of the infinite hierarchy are

$$
i u_{t}+\sum_{n=1}^{\infty}\left(\alpha_{2 n} K_{2 n}-i \alpha_{2 n+1} K_{2 n+1}\right)=0 .
$$

The NLSE is obtained from (25) when all real coefficients except $\alpha_{2}$ are zero. Thus,

$$
i u_{t}+\alpha_{2} K_{2}=0 \text {, }
$$

where the functional $K_{2}=u_{x x}+2 u|u|^{2}$.

If $u(x, t)$ in (26) is real, this gives the real form $D_{2}$ of the functional $K_{2}$,

$$
D_{2} \equiv u_{x x}+2 u^{3}
$$

We can take the derivative of $D_{2}$ with respect to $x$

$$
M_{3}=\frac{\partial D_{2}}{\partial x}=u_{x x x}+6 u_{x} u^{2}
$$

to get the mKdV equation,

$$
i u_{t}-i \alpha_{3} M_{3}=0 .
$$

From [9], we have

$$
K_{3}=u_{x x x}+6 u_{x}|u|^{2} .
$$

Plainly, $S_{3}$ and $K_{3}$ are related through

$S_{3}-K_{3}=\left(\frac{3}{2} u\right)\left|\begin{array}{cc}u & u^{*} \\ u_{x} & u_{x}^{*}\end{array}\right|=\frac{3}{2} u\left(u u_{x}^{*}-u^{*} u_{x}\right)=\frac{3}{2} u W_{1}$,

where the determinant $W_{1}$ is defined as

$$
W_{1}=\left|\begin{array}{cc}
u & u^{*} \\
u_{x} & u_{x}^{*}
\end{array}\right|
$$

If $u$ is real, this determinant is zero.

For $u$ real, the SSE

$$
u_{t}-\alpha_{3}\left[u_{x x x}+\frac{3}{2}\left(|u|^{2}\right)_{x} u+3|u|^{2} u_{x}\right]=0
$$

reduces to the basic mKdV equation

$$
u_{t}-\alpha_{3}\left(u_{3 x}+6 u^{2} u_{x}\right)=0 \text {, }
$$

i.e. $u_{t}-\alpha_{3} M_{3}=0$, where $M_{3}=u_{3 x}+6 u^{2} u_{x}$. The Hirota functional, $K_{3}=u_{x x x}+6|u|^{2} u_{x}$, also reduces to $M_{3}$ for $u$ real.

Converting (13) to its $b^{2}=2$ form, we get

$$
\begin{aligned}
S_{4}= & 3 u_{x x}^{*} u^{2}+6 u|u|^{4}+6\left|u_{x}\right|^{2} u \\
& +7|u|^{2} u_{x x}+4 u^{*} u_{x}^{2}+u_{x x x x} .
\end{aligned}
$$


It is clear that (30) has the form of the fourth member of the Hirota (NLS) hierarchy, which is known as the LPD equation, but has different coefficients. Now, from [9]:

$$
\begin{aligned}
K_{4}= & 2 u_{x x}^{*} u^{2}+6 u|u|^{4}+4\left|u_{x}\right|^{2} u \\
& +8|u|^{2} u_{x x}+6 u^{*} u_{x}^{2}+u_{x x x x} .
\end{aligned}
$$

The difference between $S_{4}$ and $K_{4}$ is

$$
S_{4}-K_{4}=2\left|u_{x}\right|^{2} u-2 u^{*} u_{x}^{2}+u_{x x}^{*} u^{2}-|u|^{2} u_{x x},
$$

so the two fourth-order equations differ only by two terms:

$$
\begin{aligned}
u\left(S_{4}-K_{4}\right) & =\left|\begin{array}{cc}
u & u^{*} \\
\frac{\partial}{\partial x}\left(u_{x} u^{2}\right) & \frac{\partial}{\partial x}\left(u_{x}^{*} u^{2}\right)
\end{array}\right| \\
& =u \frac{\partial}{\partial x}\left(u_{x}^{*} u^{2}\right)-u^{*} \frac{\partial}{\partial x}\left(u_{x} u^{2}\right),
\end{aligned}
$$

so

$$
S_{4}-K_{4}=u W_{2}+2 u_{x} W_{1},
$$

where

$$
W_{2}=\left|\begin{array}{cc}
u & u^{*} \\
u_{2 x} & u_{2 x}^{*}
\end{array}\right| \text {. }
$$

If $u$ is real, then $S_{4}-K_{4}$ is also zero.

If we take $u$ to be real, then we clearly have $S_{4}-K_{4}=0$, and we get

$$
S_{4}=K_{4}=10 u_{x x} u^{2}+6 u^{5}+10 u u_{x}^{2}+u_{x x x x} \equiv D_{4} .
$$

Now $D_{4}$ is the real form, which can be used to obtain the fifth-order mKdV equation [40],

$$
u_{t}-\alpha_{5} M_{5}=0 .
$$

Thus,

$$
\begin{aligned}
\frac{\partial D_{4}}{\partial x} & =30 u_{x} u^{4}+10 u_{x x x} u^{2}+40 u_{x} u_{x x} u+10 u_{x}^{3}+u_{5 x} \\
& =M_{5} .
\end{aligned}
$$

This is the same as the functional $M_{5}$ found by other means. It is also the same as (22) of the real-valued SSE hierarchy, $S_{5}$, found here, and it is the same as the realvalued form of $K_{5}$ found in [9].

Indeed, from [9], we have

$$
\begin{aligned}
K_{5}= & u_{5 x}+10|u|^{2} u_{x x x}+10\left(u\left|u_{x}\right|^{2}\right)_{x} \\
& +20 u^{*} u_{x} u_{x x}+30|u|^{4} u_{x} .
\end{aligned}
$$

We furthermore find that $S_{5}$ and $K_{5}$ are related as follows:

$$
\begin{aligned}
S_{5}-K_{5}= & \frac{5}{2} u W_{3}+\frac{5}{2} u_{x} W_{2} \\
& +\left(\frac{5}{2} u_{2 x}+10 u|u|^{2}\right) W_{1},
\end{aligned}
$$

where

$$
W_{3}=\left|\begin{array}{cc}
u & u^{*} \\
u_{3 x} & u_{3 x}^{*}
\end{array}\right|,
$$

and $W_{1}$ and $W_{2}$ defined above. We can generalise these definitions as follows:

$$
W_{j}=\left|\begin{array}{cc}
u & u^{*} \\
u_{j x} & u_{j x}^{*}
\end{array}\right|,
$$

so that the $W_{j}$ determinant involves the $j^{\text {th }}$ derivatives of $u$ and its complex conjugate, $u^{*}$. When $u$ is real, then each $W_{j}$ is zero. If $u$ is real, then $S_{5}-K_{5}$ is also zero, so $S_{5}=K_{5}=M_{5}$, as noted above.

The real scaled form of $S_{6}$ from this present work is

$$
\begin{aligned}
S_{6}= & 20 u^{7}+70 u^{4} u_{x x}+140 u^{3} u_{x}^{2}+14 u^{2} u_{x x x x} \\
& +14 u\left(4 u_{x} u_{x x x}+3 u_{x x}^{2}\right)+70 u_{x}^{2} u_{x x}+u_{6 x} .
\end{aligned}
$$

The real form of $K_{6}$, given in [9], is exactly the same as (35). These two functionals are clearly identical, and we label them $D_{6}$. Now, we can obtain the seventh-order mKdV equation, $u_{t}-\alpha_{7} M_{7}=0$, where

$$
\begin{aligned}
\frac{\partial D_{6}}{\partial x}= & u_{7 x}+14 u^{2}\left(u_{5 x}+30 u_{x}^{3}\right) \\
& +140 u^{6} u_{x}+70 u^{4} u_{x x x}+560 u^{3} u_{x} u_{x x}+ \\
& +28 u\left(3 u_{x} u_{x x x x}+5 u_{x x} u_{x x x}\right) \\
& +182 u_{x} u_{x x}^{2}+126 u_{x}^{2} u_{x x x} \\
= & M_{7}
\end{aligned}
$$

This is the seventh-order mKdV functional. It is the same as the real form of $K_{7}$. The forms of the mKdV functionals, $M_{2 j+1}$, agree with those found from the recursion operator.

Also, from [9], we have

$$
\begin{aligned}
K_{6}= & u_{6 x}+2\left[30 u^{*}\left|u_{x}\right|^{2}+25\left(u^{*}\right)^{2} u_{x x}+u_{x x x x}^{*}\right] u^{2} \\
& +u\left[12 u^{*} u_{x x x x}+8 u_{x} u_{x x x}^{*}+22\left|u_{x x}\right|^{2}\right]
\end{aligned}
$$




$$
\begin{aligned}
& +u\left[18 u_{x x x} u_{x}^{*}+70\left(u^{*}\right)^{2} u_{x}^{2}\right] \\
& +20\left(u_{x}\right)^{2} u_{x x}^{*}+10 u_{x}\left[5 u_{x x} u_{x}^{*}+3 u^{*} u_{x x x}\right] \\
& +20 u^{*} u_{x x}^{2}+10 u^{3}\left[\left(u_{x}^{*}\right)^{2}+2 u^{*} u_{x x}^{*}\right] \\
& +20 u|u|^{6}
\end{aligned}
$$

We furthermore find that $S_{6}$ and $K_{6}$ are related as follows:

$$
\begin{aligned}
S_{6}-K_{6}= & 2 u W_{4}+8 u_{x} W_{3} \\
& +\frac{1}{2}\left(9 u_{2 x}+15 u|u|^{2}+\frac{5}{u} u_{x}^{2}\right) W_{2} \\
& +\frac{1}{4}\left[15 u^{2} u_{x}^{*}+105|u|^{2} u_{x}\right. \\
& \left.-\frac{10}{u} u_{x} u_{x x}+2 u_{x x x}\right] W_{1},
\end{aligned}
$$

\begin{tabular}{|c|c|c|c|c|c|c|}
\hline \multicolumn{2}{|c|}{ density } & \multicolumn{2}{|c|}{$S_{i}$ (complex) } & real form & \multicolumn{2}{|c|}{ NLS(complex) } \\
\hline \multirow[t]{3}{*}{$\overline{H_{3}}$} & $\longrightarrow$ & $S_{2}$ & $\longrightarrow$ & $D_{2}$ & $\longleftarrow$ & $K_{2}[\mathrm{NLS}]=S_{2}$ \\
\hline & & & & $\downarrow \frac{\partial}{\partial x}$ & & \\
\hline & & $5_{3}[\mathrm{SSE}]$ & $\longrightarrow$ & $M_{3}$ & $\longleftarrow$ & $K_{3}[$ Hirota $]$ \\
\hline \multirow[t]{3}{*}{$\mathrm{H}_{5}$} & $\longrightarrow$ & $S_{4}$ & $\longrightarrow$ & $D_{4}$ & $\longleftarrow$ & $K_{4}[\mathrm{LPD}]$ \\
\hline & & & & $\downarrow \frac{\partial}{\partial x}$ & & \\
\hline & & $S_{5}$ & $\longrightarrow$ & $M_{5}$ & $\longleftarrow$ & $K_{5}$ [quintic] \\
\hline \multirow[t]{3}{*}{$H_{7}$} & $\longrightarrow$ & $S_{6}$ & $\longrightarrow$ & $D_{6}$ & $\longleftarrow$ & $K_{6}[$ sextic $]$ \\
\hline & & & & $\downarrow \frac{\partial}{\partial x}$ & & \\
\hline & & $S_{7}$ & $\longrightarrow$ & $M_{7}$ & $\longleftarrow$ & $\kappa_{7}[$ heptic] \\
\hline \multirow[t]{2}{*}{$H_{2 j+1}$} & $+1 \longrightarrow$ & $\Rightarrow \quad S_{2 j}$ & $\longrightarrow$ & $D_{2 j}$ & $\longleftarrow$ & $K_{2 j}$ \\
\hline & & $S_{2 j+1}$ & $\longrightarrow$ & $M_{2 j+1}$ & $\longleftarrow \quad k$ & $2 j+1$ \\
\hline
\end{tabular}

If $u$ is real, then each $W_{j}$ is zero, and thus, $S_{6}=K_{6}$.

In the expressions $S_{j}-K_{j}$, the first term is proportional to $u W_{j-2}$, while the second term is proportional

Table 1: Relations between hierarchies, summarising the results of Section 4.

Here $H_{2 j+1}$ [(7), (13) and (15)] indicates invariant density of basic SSE, $K_{j}$ indicates $j^{\text {th }}$-order functional of NLS hierarchy, $S_{j}$ indicates $j^{\text {th }}$-order functional of the SSE hierarchy (newly presented here), $M_{j}$ (33) indicates $j^{\text {th }}$-order functional of mKdV hierarchy. If the function $u$ is specified as being real, then both $K_{2 j}$ and $S_{2 j}$ reduce to the same functional, viz. $D_{2 j}$ [(27), (32) and (35)] for each $j$. Each vertical arrow indicates $\frac{\partial}{\partial x}$, so that $\frac{\partial D_{2 j}}{\partial x}=M_{2 j+1}$; for example, see (28), (33) and (36). to $u_{x} W_{j-3}$. Of course, when the $K_{j}$ and differences are known, the set $S_{j}$ can be generated. So, the pattern of these hierarchies is now more evident. The results of this section are illustrated schematically in Table 1.

\section{Conclusions}

Using densities, we have derived a new general multiparameter equation that contains, as particular cases, the Sasa-Satsuma and mKdV hierarchies of equations. Real arbitrary parameters in this equation allow one to select any particular equation of these hierarchies and any combination of them. While we have presented the operators involved in this equation in explicit forms up to order 6, the technique given here in principle allows one to conveniently obtain the equation of any even order.

This generalised SSE (2), in addition to the generalised Hirota equation [8, 9], will be useful in improving the accuracy of modelling solitons, breathers, and rogue waves and even turbulent phenomena in integrable systems [41]. It will allow inclusion of higher-order effects into mathematical modelling of systems using the form with any number of real parameters to control the dynamics being investigated.

Finding solutions of the original SSE is not easy [42-45]. It would be even more difficult to find them for the whole generalised (2). Nevertheless, integrability means there is a way to find solutions in analytic form. An example of a soliton solution for the whole infinite equation has been given in [29]. We believe this work can be continued, and, in a few years, we will be able to see more detailed analysis along this path. This immense work needs collective efforts.

Acknowledgements: N. Akhmediev is a recipient of the Alexander von Humboldt Award. The authors gratefully acknowledge the support from the Australian Research Council (Discovery Projects DP140100265 and DP150102057) and from Volkswagen Stiftung. U. Bandelow acknowledges support by the German Research Foundation in the framework of the Collaborative Research Center 787 "Semiconductor Nanophotonics" under project B5.

\section{References}

[1] S. A. R. Horsley, J. Opt. 18, 085104 (2016).

[2] S. Ghosh, B. Talukdar, and J. Shamanna, Czechoslovak J. Phys. 53, 425 (2003).

[3] A. Kundu, Symmetry Integr. Geom. 2, 078 (2006). 
[4] D. Gieseker, Commun. Math. Phys. 181, 587 (1996).

[5] N. Joshi, Publ. RIMS, Kyoto Univ. 40, 1039 (2004).

[6] L. D. Faddeev and L. A. Takhtajan, Hamiltonian Methods in the Theory of Solitons, Springer-Verlag, Berlin, Heidelberg 1987.

[7] K. Porsezian, M. Daniel, and M. Lakshmanan, J. Math. Phys. 33, 1807 (1992).

[8] D. J. Kedziora, A. Ankiewicz, A. Chowdury, and N. Akhmediev, Chaos 25, 103114 (2015).

[9] A. Ankiewicz, D. J. Kedziora, A. Chowdury, U. Bandelow, and N. Akhmediev, Phys. Rev. E 93, 012206 (2016).

[10] C. S. Gardner, J. M. Greene, M. D. Kruskal, and R. M. Miura, Phys. Rev. Lett. 19, 1095 (1967).

[11] V. E. Zakharov and A. B. Shabat, Sov. Phys. JETP 34, 62 (1972)

[12] A. Blanco-Redondo, C. Husko, D. Eades, Y. Zhang, J. Li, et al., Nat. Commun. 5, 3160 (2014).

[13] F. M. Mitschke and L. F. Mollenauer, Opt. Lett. 11, 659 (1986).

[14] D. Anderson, M. Desaix, M. Lisak, and M. L. Quiroga-Teixeiro, J. Opt. Soc. Am. B 9, 1358 (1992).

[15] A. Hasegawa and F. Tappert, Appl. Phys. Lett. 23, 142 (1973).

[16] S. B. Cavalcanti, J. C. Cressoni, H. R. da Cruz, and A. S. Gouveia-Neto, Phys. Rev. A 43, 6162 (1991).

[17] M. Trippenbach and Y. B. Band, Phys. Rev. A 57, 4791 (1991).

[18] M. J. Potasek, Phys. Lett. A 60, 449 (1991).

[19] H. C. Yuen and B. M. Lake, Adv. Appl. Mech. 22, 67 (1982).

[20] K. B. Dysthe, Proc. R. Soc. Lond. A 369, 105 (1979)

[21] Y. V. Sedletskii, Sov. Phys. JETP 97, 180 (2003) [Translated from Zh. Eksper. Teor. Fiziki. 124, 200 (2003)].

[22] A. V. Slunyaev, Sov. Phys. JETP 101, 926 (2005) [Translated from Zh. Eksper. Teor. Fiziki 128, 1061 (2005)].

[23] F. Baronio, M. Conforti, A. Degasperis, and S. Lombardo, Phys. Rev. Lett. 111, 114101 (2013).

[24] F. Baronio, A. Degasperis, M. Conforti, and S. Wabnitz, Phys. Rev. Lett. 109, 044102 (2012).

[25] Y. Ohta and J. K. Yang, Phys. Rev. E 86, 036604 (2012).
[26] Y. Ohta and J. K. Yang, J. Phys. A Math. Theor. 46, 105202 (2013).

[27] F. Baronio, Opt. Lett. 42, 1756 (2017).

[28] G. Biondini, K. Maruno, M. Oikawa, and H. Tsuji, Stud. Appl. Math. 123, 375 (2009)

[29] U. Bandelow, A. Ankiewicz, Sh. Amiranashvili, and N. Akhmediev, Chaos (Interdis. J. Nonlinear Sci.) 28, 053108 (2018); doi: $10.1063 / 1.5030604$.

[30] R. Hirota, J. Math. Phys. 14, 805 (1973).

[31] M. Lakshmanan, K. Porsezian, and M. Daniel, Phys. Lett. A 133, 483 (1988).

[32] S. M. Hoseini and T. R. Marchant, Wave Motion 44, 92 (2006).

[33] A. Chowdury, D. J. Kedziora, A. Ankiewicz, and N. Akhmediev, Phys. Rev. E 91, 032928 (2015).

[34] N. Sasa and J. Satsuma, J. Phys. Soc. Jpn. 60, 409 (1991).

[35] C. Gilson, J. Hientarinta, J. Nimmo, and Y. Ohta, Phys. Rev. E 68, 016614 (2003).

[36] D. Mihalache, L. Torner, F. Moldoveanu, N. C. Panoiu, and N. Truta, J. Phys. A Math. Gen. 26, L757 (1993).

[37] N. Akhmediev and A. Ankiewicz, Solitons, Nonlinear Pulses and Beams, Chapman \& Hall, London 1997.

[38] S. Ghosh, A. Kundu, and S. Nandy, J. Math. Phys. 40, 1993 (1999).

[39] P. A. Clarkson, N. Joshi, and M. Mazzocco, Sem. Congr. 14, 53 (2006).

[40] Y. Matsuno, J. Phys. Soc. Jpn. 49, 787 (1980).

[41] N. Akhmediev, J. M. Soto-Crespo, and N. Devine, Phys. Rev. E 94, 022212 (2016).

[42] D. Mihalache, N. C. Panoiu, F. Moldoveanu, and D.-M. Baboiu, J. Phys. A Math. Gen. 27, 6177 (1994).

[43] D. Mihalache, L. Torner, F. Moldoveanu, N. C. Panoiu, and N. Truta, Phys. Rev. E 48, 4699 (1993).

[44] U. Bandelow and N. Akhmediev, Phys. Lett. A 376, 1558 (2012).

[45] O. C. Wright III, Chaos Soliton Fract 33, 374 (2007). 